hep-ph/9712419

Freiburg-THEP $97 / 28$

November 1997

\title{
Two-loop large Higgs mass corrections to electroweak gauge boson quartic couplings
}

\author{
V. Borodulin \\ Institute for High Energy Physics \\ Protvino, Moscow Region 142284, Russia \\ G. Jikia \\ Albert-Ludwigs-Universität Freiburg, Fakultät für Physik \\ Hermann-Herder Str.3, D-79104 Freiburg, Germany
}

\begin{abstract}
The two-loop corrections to the electroweak gauge boson quartic couplings, growing quadratically with the Higgs boson mass, are calculated in the Standard Model in the limit of large Higgs mass. The corrections to $W W W W, W W Z Z$ and $Z Z Z Z$ four-vertices are found to be an order of magnitude larger than the two-loop $m_{H}^{2}$ corrections to light fermion and triple gauge boson vertices. For a heavy Higgs boson with a mass around $1 \mathrm{TeV}$ the corrections are at the several percent level and in principle could be observed experimentally.
\end{abstract}




\section{Introduction}

The remarkable precision of the electroweak experimental data [1, 2] makes it possible to test the predictions of the Standard Model $(\mathcal{S M})$ at the quantum loop level. After the successful prediction of the top-quark mass from the $m_{t}^{2}$ one-loop electroweak radiative corrections and the actual observation of the top quark signal at the Tevatron, the mechanism of the spontaneous electroweak symmetry breaking, connected to the existence of the Higgs boson in the $\mathcal{S M}$, remains the last untested property of the $\mathcal{S M}$. Electroweak observables are influenced also by the presence of the Higgs boson, but contrary to the $m_{t}^{2}$ dependence at the one-loop level they depend only logarithmically on the Higgs boson mass. From the high-precision data at LEP, SLC and the Tevatron an upper limit of $m_{H}<430 \mathrm{GeV}$ has been derived at the $95 \%$ confidence level [1,2]. This bound is not very sharp however. It is known, that excluding one or two observables from the global $\mathcal{S} \mathcal{M}$ fit weakens the bound significantly [3, 国. The reason is that the restrictive upper bound on $m_{H}$ depends crucially on the world average of the effective electroweak mixing angle $s_{\text {eff }}^{2}$, for which the experimental average values from LEP and SLC differ by 2.9 standard deviations [1,2]. As an illustration of this situation it has been shown recently [ [4], that employing only the LEP average one obtains a 95\%C.L. upper bound for the Higgs mass larger than $800 \mathrm{GeV}$, while using the SLD value alone the corresponding bound is approximately $80 \mathrm{GeV}$. In a conservative conclusion the experimental limit may therefore be interpreted in the $\mathcal{S} \mathcal{M}$ as an indication for a scale $m_{H} \leq \mathcal{O}(1) \mathrm{TeV}$.

In order to evaluate the heavy Higgs signal at high energy and estimate the region of applicability of the perturbation theory, the leading two-loop corrections of enhanced electroweak strength were under intense study. In particular, the high energy weak-boson scattering in the limit $s \gg m_{H}^{2} \gg M_{W}^{2}$ [0], renormalization constants at the Higgs pole [6] 9], corrections to the partial widths of the Higgs boson decay to pairs of fermions [8, 10, 11] and intermediate vector bosons [12, 13], corrections to the heavy Higgs line shape at LHC [14] and $\mu^{+} \mu^{-}$collider 15] have been calculated at two-loops to leading order in $m_{H}^{2}$. In addition, recently nonperturbative next-to-leading corrections to the Higgs propagator and Higgs boson parameters have been calculated in the $1 / N$ expansion [16].

However, if the Higgs boson is really heavy the study of its indirect effects at the quantum loop level at energies much smaller than $m_{H}$ will be one of the most important goals for the future experiments. As it was already mentioned, although at the one-loop level one might expect contributions to the $W, Z$ vector boson mass shifts to be proportional to $m_{H}^{2}$, for $m_{H} \gg M_{W}$ the leading terms cancel out and only the $\log m_{H}^{2}$ dependence survives [17]. This has been referred to by Veltman as a screening theorem [17]. Motivated partly by this phenomenon, van der Bij and Veltman calculated the two-loop large Higgs mass corrections to the $\rho$-parameter [18] and to vector boson masses [19]. These results were verified in Ref. [20]. In these papers it has been shown that, although some of the diagrams are proportional to $m_{H}^{4}$, they cancel out in observable corrections, leaving only terms proportional to $m_{H}^{2}$. It has been proven lately to all orders that in the $\mathcal{S} \mathcal{M}$ vector propagators can contribute to low energy observable quantities at most $\left(m_{H}^{2}\right)^{(L-1)}$ dependence on the Higgs boson mass at the $L$-loop level for $m_{H} \gg M_{W}$ [21]. These two-loop large Higgs mass calculations were extended by van der Bij to the case of the triple vector boson couplings [22]. No cancellations of the leading terms happen in this case and the two-loop corrections growing like $m_{H}^{2}$ were found in agreement with the naive power counting arguments. The same power counting shows, that only vertex functions with maximally four vector boson external legs can have two-loop large Higgs mass corrections proportional to $m_{H}^{2}$, while for five and higher point vertex functions no power growth of the two-loop corrections with the Higgs mass is possible.

The main objective of the present paper is to complete these calculations and obtain the analytical expressions for the two-loop $m_{H}^{2}$ corrections to quartic electroweak gauge boson couplings in the $\mathcal{S} \mathcal{M}$ in the limit $m_{H} \gg M_{W}$ at low energy $E \ll m_{H}$, and thereby to obtain the complete two-loop low energy $\mathcal{S} \mathcal{M}$ effective action $\Gamma_{\text {eff }}$ to order $m_{H}^{2}$.

The paper is organized as follows: after some preliminary discussion of the calculational framework in Section 2, we describe the details of the calculation in Section 3. In Section 4 all the analytical results are presented. Section 5, where numerical results are given, is devoted to a discussion of the implications for the physical processes. Section 6 contains our conclusions. 


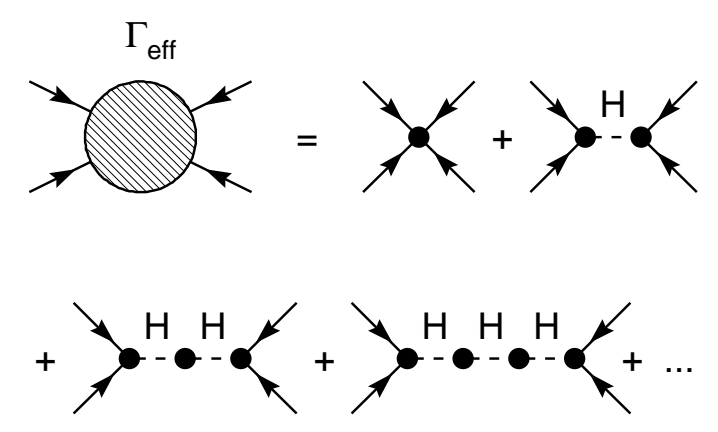

Figure 1: One particle irreducible and Higgs reducible graphs contributing to low energy quartic vector boson vertex. Bold blobs denote the one particle irreducible four-, three- and two-point vertex functions.

\section{Calculational framework}

The calculations are done for the $\mathcal{S M}$ in the 't Hooft-Feynman gauge. We neglect fermion masses, so only bosonic loop diagrams contribute [18]. In order to calculate the four vector boson vertex function contribution to the low energy effective action $\Gamma_{\text {eff }}$ one has to take into account both one-particle irreducible (OPI) fourvertex graphs and one particle Higgs reducible graphs with four external vector particles, as shown in Fig. 1. Since the Higgs self energy at two-loop (one-loop) level is proportional to $m_{H}^{6}\left(m_{H}^{4}\right)$ and $H W^{+} W^{-}, H Z Z$, $H \gamma \gamma, H \gamma Z$ triple vertices at two-loop (one-loop) level grow like $m_{H}^{4}\left(m_{H}^{2}\right)$, these Higgs reducible graphs do contribute to the leading $m_{H}^{2}$ dependence inspite of the $1 / m_{H}^{2}$ suppression due to the Higgs propagators. No one-particle reducible graphs contribute to two- and three-point vertices and at two-loop order only quartic $W^{+} W^{-} W^{+} W^{-}, W^{+} W^{-} Z Z$ and $Z Z Z Z$ vertices include a $m_{H}^{2}$ contribution from two-loop Higgs self energy. Due to this fact, these vertices play a special role as a probe of the mechanism of the electroweak symmetry breaking sector. Since $H \gamma \gamma, H \gamma Z$ vertices are equal to zero at tree-level, although the Higgs reducible graphs also contribute to the $\gamma Z W^{+} W^{-}, \gamma \gamma W^{+} W^{-}, \gamma \gamma Z Z$ and other four-vertices with at least one external photon, only one-loop Higgs self energy graphs contribute.

The calculations are done in the on-mass-shell renormalization scheme [23]. In this scheme all counterterms are fixed uniquely by the requirements that the pole positions of the Higgs, $W$ and $Z$ boson propagators coincide with their physical masses, the corresponding residues are normalized to unity and electric charge is renormalized to give the observable value at low energy.

Since a heavy Higgs boson is a highly unstable particle there is an ambiguity in the definition of its mass 24,25]. If the exact Higgs propagator is defined by

$$
\Delta_{H}(s)=\frac{1}{s-m_{H}^{2}-\Sigma(s)},
$$

one can define the Higgs boson mass either as a zero of the real part of the inverse propagator

$$
\operatorname{Re}\left(\Delta_{H}^{-1}\left(m_{H}^{2}\right)\right)=0
$$

or as a real part of the complex pole

$$
\Delta_{H}^{-1}\left(m_{H}^{2}-i m_{H} \Gamma_{H}\right)=0 .
$$

Only the complex pole mass value was shown to be gauge invariant 24, 25]. In our case, however, since the leading $m_{H}^{2}$ term vanishes for the derivative of the imaginary part of Higgs self energy at the one-loop level

$$
\operatorname{Im}\left(\Sigma^{1-l o o p}\right)^{\prime}\left(m_{H}^{2}\right)=0
$$

both definitions give the same value of the Higgs boson mass at the two-loop order. The necessary Higgs wave function renormalization constant and Higgs mass counterterm were calculated analytically in our paper [8] 
in complete agreement with the partly numerical results [6, 0, 10, 11. The two-loop wave function and mass counterterms for electroweak gauge bosons are known since papers 18,19 .

As was mentioned in Ref. 8] there are two equivalent definitions of the on-mass-shell renormalization scheme at two-loop order: the standard one, when the one-loop counterterms are calculated including terms of $\mathcal{O}(\epsilon)$ order, since terms proportional to $\epsilon$ can combine with $1 / \epsilon$ poles at the two-loop level to give a finite contribution, and a modified one, when one-loop counterterms are calculated only to $\mathcal{O}\left(\epsilon^{0}\right)$ order. These two schemes are equivalent because the account of finite contributions coming from the combination of $\mathcal{O}(\epsilon)$ oneloop counterterms with $1 / \epsilon$ overall divergence just redefines the finite parts of the two-loop counterterms. But due to tadpole diagrams special care should be taken using the modified scheme. Given the part of the $\mathcal{S M}$ Lagrangian describing the Higgs scalar sector

$$
\begin{aligned}
\mathcal{L} & =\frac{1}{2} \partial_{\mu} H_{0} \partial^{\mu} H_{0}+\frac{1}{2} \partial_{\mu} z_{0} \partial^{\mu} z_{0}+\partial_{\mu} w_{0}^{+} \partial^{\mu} w_{0}^{-} \\
& -\frac{m_{H 0}^{2}}{2 v_{0}^{2}}\left(w_{0}^{+} w_{0}^{-}+\frac{1}{2} z_{0}^{2}+\frac{1}{2} H_{0}^{2}+v_{0} H_{0}+\frac{1}{2} \delta v^{2}\right)^{2},
\end{aligned}
$$

one can choose the tadpole counterterm $\delta v^{2}$ in such a way, that a Higgs field vacuum expectation value is equal to $v_{0}$

$$
v_{0}=\frac{2 M_{W_{0}}}{g}
$$

to all orders, so that tadpole diagrams and corresponding counterterms always cancel out and one can just ignore all the tadpole diagrams altogether. If all the particles are massive it is enough to require the cancellation of the one-loop tadpole diagrams with $\delta v^{2}$ counterterm to order $\mathcal{O}\left(\epsilon^{0}\right)$, because for any two-loop Higgs reducible diagram with one-loop tadpole the one-loop subdiagram excluding the tadpole is finite when summed with the corresponding counterterms and terms proportional to $\epsilon$ do not contribute. This however is only true if this subdiagram is infrared finite, since counterterms cancel only ultraviolet divergences. If infrared $1 / \epsilon$ poles are present, they can combine with $\mathcal{O}(\epsilon)$ terms resulting from incomplete tadpole and $\delta v^{2}$ counterterm cancellation to produce finite nonzero contribution. And this really happens for the $W^{+} W^{-} W^{+} W^{-}$vertex. In order to still validate the neglect of the tadpole diagrams one should either take into account the one-loop tadpole $\delta v^{2}$ counterterm to order $\mathcal{O}(\epsilon)$ or regulate infrared divergences introducing an infinitesimal photon mass $\lambda$. Coincidence of the renormalized vertices in both schemes was one of the consistency checks of the calculation.

\section{The calculation}

The two-loop topologies and one-loop topologies with counterterm insertions contributing to OPI four-, three-, and two-point vertex functions are shown in Fig. 2. The numbers in parentheses show the total number of corresponding topologies, the external lines are assumed to be topologically different.

The evaluation of these graphs proceeds in a number of steps.

The first step consists of the generation of all topologically distinct graphs followed by the assignment of particles to the internal lines according to the $\mathcal{S} \mathcal{M}$ Feynman rules. During this procedure duplicated graphs are eliminated and symmetry factors are evaluated. The total number of graphs generated is quite large. E.g., for irreducible $W^{+} W^{-} W^{+} W^{-}$vertex 84698 two-loop diagrams and 2424 one-loop diagrams contribute, but of course, not all of them produce the $m_{H}^{2}$ dependence.

Since contributions growing with the Higgs mass at energies much smaller than $m_{H}$ are described by the low energy effective action $\Gamma_{\text {eff }}$ given by a set of local operators, containing only a finite number of derivatives, these operators can be evaluated making Taylor expansion around zero in the external momenta. According to power counting only the first term of the expansion has a leading $m_{H}^{2}$ dependence for four-point vertex functions with physical vector bosons as external particles. For these vertices it is enough just to set all external momenta equal to zero. However, for three- and two-point functions and four-point functions with unphysical Nambu-Goldstone scalar external particles one should keep more terms in the Taylor expansion. So at the second step explicit expressions for all the vertices are substituted and when necessary Taylor 

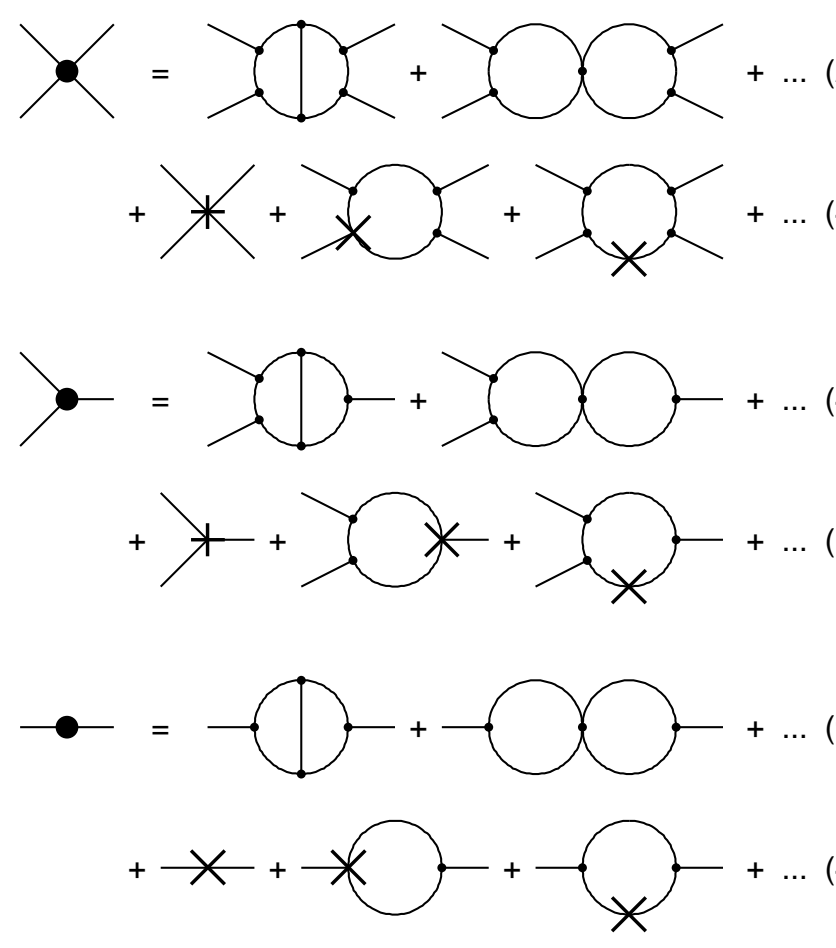

Figure 2: One-particle irreducible two- and one-loop topologies.

expansions are made. After this (trivial for four-vertices with physical external particles) step one is left with the sum of the two-loop vacuum diagrams.

The third step consists of the elimination of diagrams that do not grow like a positive power of the Higgs mass. A procedure based on the so-called asymptotic operation method 26] is used to count the maximal power of growth with $m_{H}$ corresponding to a given vacuum integral.

The fourth step consists of the reduction of the remaining tensor vacuum integrals to scalar ones, removing scalar products of loop momenta in numerators and of the splitting of propagators with the same momentum but different masses via partial fractioning relations. After that everything is expressed in terms of the scalar vacuum two-loop integrals of the following form

$$
\begin{aligned}
& J\left(n_{1} m_{1}^{2}, n_{2} m_{2}^{2}, n_{3} m_{3}^{2}\right)= \\
& -\frac{1}{\pi^{4}} \int D^{(d)} P D^{(d)} Q\left(P^{2}-m_{1}^{2}\right)^{-n_{1}}\left(Q^{2}-m_{2}^{2}\right)^{-n_{2}}\left((P-Q)^{2}-m_{3}^{2}\right)^{-n_{3}} .
\end{aligned}
$$

At step five the integrals (3.1) are reduced using the recurrence relations based on the integration by parts technique [27] to the integrals of the same form (3.1) with all the powers $n_{i} \leq 1$.

And at sixth step the remaining scalar vacuum integrals are calculated using if necessary the asymptotic expansions method [26], if both heavy Higgs boson mass $m_{H}$ and light $M_{W}, M_{Z}$ masses enter the integral (3.1).

The procedure for the evaluation of the one-loop diagrams with the counterterm insertions is essentially the same, but of course much simpler.

Calculated OPI graphs are then substituted into the Higgs reducible graphs, as shown in Fig. 1, to obtain corresponding operators of the low energy effective action $\Gamma_{\text {eff }}$. As a result all the amplitudes are expressed in terms of transcendental functions $\zeta(3)$ and the maximal value of the Clausen function $\mathrm{Cl}(\pi / 3)$.

In order to check the results obtained in addition to obvious consistency checks like the finiteness of the renormalized results and symmetry properties, the other cross checks were also done. As was mentioned at 
the end of Section 2, we have done the calculations in two different renormalization schemes: one with zero photon mass and one-loop counterterms calculated to order $\mathcal{O}(\epsilon)$ and the other with small nonzero photon mass $\lambda$ and one-loop counterterms calculated to order $\mathcal{O}\left(\epsilon^{0}\right)$. The results for the renormalized effective action were found to be identical. Moreover, some individual diagrams are infrared divergent and since there are no tree-level or one-loop vertices with the emission of additional photon, which would grow like $m_{H}^{2}$, this infrared divergences should be canceled out in the sum of all contributing diagrams. In the second renormalization scheme it is possible to explicitly observe that all singular $\operatorname{logarithms} \log \lambda^{2}$ really cancel out.

As a last verification of the calculation the Ward identities in the form, which is a basis of the equivalence theorem [28],

$$
\begin{aligned}
& \left\langle 0\left|T\left[\left(\partial^{\mu} W_{\mu}^{+}\left(x_{1}\right)+M_{W} w^{+}\left(x_{1}\right)\right) W_{\nu}^{-}\left(x_{2}\right) W_{\alpha}^{+}\left(x_{3}\right) W_{\beta}^{-}\left(x_{4}\right)\right]\right| 0\right\rangle_{a m p}=0 \\
& \left\langle 0\left|T\left[\left(\partial^{\mu} Z_{\mu}\left(x_{1}\right)+M_{Z} z\left(x_{1}\right)\right) Z_{\nu}\left(x_{2}\right) W_{\alpha}^{+}\left(x_{3}\right) W_{\beta}^{-}\left(x_{4}\right)\right]\right| 0\right\rangle_{a m p}=0, \quad \cdots
\end{aligned}
$$

have been checked for all the four-particle amplitudes. Here $w$ and $z$ are the unphysical Nambu-Goldstone partners of $W, Z$ bosons. In order to check the identities (3.2) all the amplitudes with three external vector bosons and one non-physical scalar were calculated. If the first external vector boson is a photon, then the identity (3.2) is just a transversality condition. The chronological product here is in fact a complete renormalized four-particle Feynman amplitude with amputated external legs, including OPI as well as oneparticle reducible graphs with light particle propagators. Usually the Ward identities (3.2) are formulated for unrenormalized fields and non-amputated external leg for $\partial^{\mu} W, w\left(\partial^{\mu} Z, z\right)$ lines [2\&], however taking into account that non-diagonal self-energies (4.15), 4.16) also satisfy corresponding Ward identities

$$
\begin{aligned}
i k^{\mu}\left\langle 0\left|T\left[W_{\mu}^{+}(k) W_{\nu}^{-}(-k)\right]\right| 0\right\rangle & =M_{W}\left\langle 0\left|T\left[w^{+}(k) W_{\nu}^{-}(-k)\right]\right| 0\right\rangle, \\
i k^{\mu}\left\langle 0\left|T\left[Z_{\mu}(k) Z_{\nu}(-k)\right]\right| 0\right\rangle & =M_{Z}\left\langle 0\left|T\left[z(k) Z_{\nu}(-k)\right]\right| 0\right\rangle
\end{aligned}
$$

one can show that the identities (3.2) are really valid to two-loop order.

\section{Analytic results}

Here we present all the terms of the two-loop low-energy effective action $\Gamma_{\text {eff }}$ to order $m_{H}^{2}$, which are necessary for the calculation of the electroweak gauge boson scattering amplitudes and amplitudes for the reactions of triple $W W Z / Z Z Z$ production at the CERN Large Hadron Collider (LHC) or the electronpositron linear collider, which are sensitive to quartic vector boson couplings:

$$
\begin{aligned}
& e e \rightarrow V V f f, \\
& e e \rightarrow V V V, \\
& p p \rightarrow V V X, \\
& p p \rightarrow V V V X,
\end{aligned}
$$

where $V=\gamma, Z$ or $W^{ \pm}$and $f=e$ or $\nu_{e}$.

The fermionic part of the effective action is defined by

$$
\begin{aligned}
\Gamma_{\text {eff }}^{f e r m i}= & e Q \bar{f} \hat{A} f+\frac{g_{\text {eff }}}{\sqrt{2}}\left(\bar{f}_{L} \hat{W}^{+} f_{L}^{\prime}+\bar{f}^{\prime}{ }_{L} \hat{W}^{-} f_{L}\right) \\
& +\bar{g}_{e f f}\left(\bar{f}_{L}\left(T_{3}-Q s_{W e f f}^{2}\right) \hat{Z} f_{L}-\bar{f}_{R} Q s_{W e f f}^{2} \hat{Z} f_{R}\right) .
\end{aligned}
$$

Here effective coupling constants are observable quantities, that are measured in the process of $\mu$ decay, in fermion scattering reactions at the $Z$ boson peak, etc. They are given by the following expressions

$$
g_{\text {eff }}=\frac{e}{s_{W}}\left\{1+\frac{e^{4}}{\left(16 \pi^{2}\right)^{2} s_{W}^{4}} \frac{m_{H}^{2}}{M_{W}^{2}}\left(-\frac{11}{64} \pi \sqrt{3}-\frac{25}{1728} \pi^{2}+\frac{49}{1152}+\frac{9}{16} C l \sqrt{3}\right)\right\}
$$




$$
\begin{aligned}
\approx & \frac{e}{s_{W}}\left(1-4.66591 \times 10^{-2} \frac{e^{4}}{\left(16 \pi^{2}\right)^{2} s_{W}^{4}} \frac{m_{H}^{2}}{M_{W}^{2}}\right) \approx \frac{e}{s_{W}}\left(1-3.15948 \times 10^{-7} \frac{m_{H}^{2}}{M_{W}^{2}}\right), \\
\bar{g}_{\text {eff }}= & \frac{e}{s_{W} c_{W}}\left\{1+\frac{e^{4}}{\left(16 \pi^{2}\right)^{2} s_{W}^{4}} \frac{m_{H}^{2}}{M_{W}^{2}}\left(\frac{1}{c_{W}^{2}}\left(\frac{9}{64} \pi \sqrt{3}+\frac{3}{64} \pi^{2}-\frac{21}{128}-\frac{9}{16} C l \sqrt{3}\right)\right.\right. \\
& \left.\left.-\frac{5}{16} \pi \sqrt{3}-\frac{53}{864} \pi^{2}+\frac{119}{576}+\frac{9}{8} C l \sqrt{3}\right)\right\} \\
\approx & \frac{e}{s_{W} c_{W}}\left(1-2.51326 \times 10^{-2} \frac{e^{4}}{\left(16 \pi^{2}\right)^{2} s_{W}^{4}} \frac{m_{H}^{2}}{M_{W}^{2}}\right) \approx \frac{e}{s_{W} c_{W}}\left(1-1.70183 \times 10^{-7} \frac{m_{H}^{2}}{M_{W}^{2}}\right), \\
s_{W \text { eff }}^{2}= & s_{W}^{2}\left\{1+\frac{e^{4}}{\left(16 \pi^{2}\right)^{2} s_{W}^{4}} \frac{m_{H}^{2}}{M_{W}^{2}}\left(+\frac{5}{16} \pi \sqrt{3}+\frac{53}{864} \pi^{2}-\frac{119}{576}-\frac{9}{8} C l \sqrt{3}\right)\right\} \\
\approx & s_{W}^{2}\left(1+0.121595 \frac{e^{4}}{\left(16 \pi^{2}\right)^{2} s_{W}^{4}} \frac{m_{H}^{2}}{M_{W}^{2}}\right) \approx s_{W}^{2}\left(1+8.23369 \times 10^{-7} \frac{m_{H}^{2}}{M_{W}^{2}}\right) .
\end{aligned}
$$

Here

$$
C l=\mathrm{Cl}\left(\frac{\pi}{3}\right)=\operatorname{Im~li}_{2}\left(e^{\frac{i \pi}{3}}\right)=1.01494160640965362502 \ldots
$$

and $c_{W}$ in the on-mass-shell scheme is defined by

$$
c_{W}=\frac{M_{W}}{M_{Z}} .
$$

As was demonstrated in Ref. [18], in the limit of vanishing fermion mass, no $m_{H}^{2}$ contributions appear for twoloop diagrams with external fermion lines with subtracted one-loop subdivergences. Thus, the corrections (4.6)-(4.8) originate only from vector boson wave function, $s_{W}, c_{W}$ and electric charge renormalizations at two-loop level. The expressions (4.6)-(4.8) are equivalent to the results of [18, 20 and for the observable quantities like $\delta \rho$ give the identical values.

The renormalized self-energies of light particles are given by

$$
\begin{aligned}
\frac{\delta \Gamma_{e f f}}{\delta W_{\mu_{1}}^{+}\left(k_{1}\right) \delta W_{\mu_{2}}^{-}\left(k_{2}\right)} & =\frac{e^{4}}{\left(16 \pi^{2}\right)^{2} s_{W}^{4}} \frac{m_{H}^{2}}{M_{W}^{2}} k_{1}^{\mu_{1}} k_{1}^{\mu_{2}}\left\{-\frac{71}{192}+\frac{11}{288} \pi^{2}\right\}, \\
\frac{\delta \Gamma_{e f f}}{\delta Z_{\mu_{1}}\left(k_{1}\right) \delta Z_{\mu_{2}}\left(k_{2}\right)} & =\frac{e^{4}}{\left(16 \pi^{2}\right)^{2} c_{W}^{2} s_{W}^{4}} \frac{m_{H}^{2}}{M_{W}^{2}} k_{1}^{\mu_{1}} k_{1}^{\mu_{2}}\left\{-\frac{71}{192}+\frac{11}{288} \pi^{2}\right\}, \\
\frac{\delta \Gamma_{e f f}}{\delta w^{+}\left(k_{1}\right) \delta w^{-}\left(k_{2}\right)} & =\frac{e^{4}}{\left(16 \pi^{2}\right)^{2} s_{W}^{4}} \frac{m_{H}^{2}}{M_{W}^{4}}\left(k_{1} \cdot k_{1}\right)^{2}\left\{-\frac{71}{192}+\frac{11}{288} \pi^{2}\right\}, \\
\frac{\delta \Gamma_{e f f}}{\delta z\left(k_{1}\right) \delta z\left(k_{2}\right)} & =\frac{e^{4}}{\left(16 \pi^{2}\right)^{2} s_{W}^{4}} \frac{m_{H}^{2}}{M_{W}^{4}}\left(k_{1} \cdot k_{1}\right)^{2}\left\{-\frac{71}{192}+\frac{11}{288} \pi^{2}\right\}, \\
\frac{\delta \Gamma_{e f f}}{\delta w^{+}\left(k_{1}\right) \delta W_{\mu_{2}}^{-}\left(k_{2}\right)} & =i \frac{e^{4}}{\left(16 \pi^{2}\right)^{2} s_{W}^{4}} \frac{m_{H}^{2}}{M_{W}^{3}} k_{1}^{\mu_{2}} k_{1} \cdot k_{1}\left\{-\frac{71}{192}+\frac{11}{288} \pi^{2}\right\}, \\
\frac{\delta \Gamma_{e f f}}{\delta z\left(k_{1}\right) \delta Z_{\mu_{2}}\left(k_{2}\right)} & =i \frac{e^{4}}{\left(16 \pi^{2}\right)^{2} s_{W}^{4} c_{W}} \frac{m_{H}^{2}}{M_{W}^{3}} k_{1}^{\mu_{2}} k_{1} \cdot k_{1}\left\{-\frac{71}{192}+\frac{11}{288} \pi^{2}\right\}, \\
\frac{\delta \Gamma_{e f f}}{\delta A_{\mu_{1}}\left(k_{1}\right) \delta A_{\mu_{2}}\left(k_{2}\right)} & =\frac{\delta \Gamma_{e f f}}{\delta A_{\mu_{1}}\left(k_{1}\right) \delta Z_{\mu_{2}}\left(k_{2}\right)}=\frac{\delta \Gamma_{e f f}}{\delta z\left(k_{1}\right) \delta A_{\mu_{2}}\left(k_{2}\right)}=0 .
\end{aligned}
$$

Since mass and residue of the transverse part of the vector boson propagators are fixed via the renormalization conditions, only the finite longitudinal structure survives in (4.11), (4.12).

Triple vertices with external physical electroweak gauge bosons and triple vertices with one external unphysical scalar, which are also needed for the calculation of the vector boson scattering amplitudes in 
't Hooft-Feynman gauge, are given by

$$
\begin{aligned}
& \frac{\delta \Gamma_{e f f}}{\delta A_{\mu_{1}}\left(k_{1}\right) \delta W_{\mu_{2}}^{+}\left(k_{2}\right) \delta W_{\mu_{3}}^{-}\left(k_{3}\right)}=\frac{e^{5}}{\left(16 \pi^{2}\right)^{2} s_{W}^{4}} \frac{m_{H}^{2}}{M_{W}^{2}}\left\{+\left(g^{\mu_{1} \mu_{2}} k_{2}^{\mu_{3}}-g^{\mu_{1} \mu_{3}} k_{3}^{\mu_{2}}\right)\left(-\frac{7}{12}+\frac{1}{18} \pi^{2}\right)\right. \\
& \left.+\left(g^{\mu_{1} \mu_{2}} k_{3}^{\mu_{3}}-g^{\mu_{1} \mu_{3}} k_{2}^{\mu_{2}}\right)\left(-\frac{41}{192}+\frac{5}{288} \pi^{2}\right)\right\} \\
& \approx \frac{e^{5}}{\left(16 \pi^{2}\right)^{2} s_{W}^{4}} \frac{m_{H}^{2}}{M_{W}^{2}}\left\{-3.50220 \times 10^{-2}\left(g^{\mu_{1} \mu_{2}} k_{2}^{\mu_{3}}-g^{\mu_{1} \mu_{3}} k_{3}^{\mu_{2}}\right)-4.21944 \times 10^{-2}\left(g^{\mu_{1} \mu_{2}} k_{3}^{\mu_{3}}-g^{\mu_{1} \mu_{3}} k_{2}^{\mu_{2}}\right)\right\}, \\
& \frac{\delta \Gamma_{e f f}}{\delta Z_{\mu_{1}}\left(k_{1}\right) \delta W_{\mu_{2}}^{+}\left(k_{2}\right) \delta W_{\mu_{3}}^{-}\left(k_{3}\right)}=-\frac{s_{W}}{c_{W}} \frac{\delta \Gamma_{e f f}}{\delta A_{\mu_{1}}\left(k_{1}\right) \delta W_{\mu_{2}}^{+}\left(k_{2}\right) \delta W_{\mu_{3}}^{-}\left(k_{3}\right)} \\
& +\frac{e^{5}}{\left(16 \pi^{2}\right)^{2} c_{W} s_{W}^{5}} \frac{m_{H}^{2}}{M_{W}^{2}}\left(g^{\mu_{1} \mu_{2}}\left(k_{2}^{\mu_{3}}-k_{1}^{\mu_{3}}\right)+g^{\mu_{1} \mu_{3}}\left(k_{1}^{\mu_{2}}-k_{3}^{\mu_{2}}\right)+g^{\mu_{2} \mu_{3}}\left(k_{3}^{\mu_{1}}-k_{2}^{\mu_{1}}\right)\right) \\
& \times\left(-\frac{217}{1152}-\frac{5}{32} \pi \sqrt{3}-\frac{5}{1728} \pi^{2}+\frac{9}{16} C l \sqrt{3}\right) \approx-\frac{s_{W}}{c_{W}} \frac{\delta \Gamma_{e f f}}{\delta A_{\mu_{1}}\left(k_{1}\right) \delta W_{\mu_{2}}^{+}\left(k_{2}\right) \delta W_{\mu_{3}}^{-}\left(k_{3}\right)} \\
& -7.83085 \times 10^{-2} \frac{e^{5}}{\left(16 \pi^{2}\right)^{2} c_{W} s_{W}^{5}} \frac{m_{H}^{2}}{M_{W}^{2}}\left(g^{\mu_{1} \mu_{2}}\left(k_{2}^{\mu_{3}}-k_{1}^{\mu_{3}}\right)+g^{\mu_{1} \mu_{3}}\left(k_{1}^{\mu_{2}}-k_{3}^{\mu_{2}}\right)+g^{\mu_{2} \mu_{3}}\left(k_{3}^{\mu_{1}}-k_{2}^{\mu_{1}}\right)\right) \text {, } \\
& \frac{\delta \Gamma_{e f f}}{\delta z\left(k_{1}\right) \delta W_{\mu_{2}}^{+}\left(k_{2}\right) \delta W_{\mu_{3}}^{-}\left(k_{3}\right)}=i \frac{e^{5}}{\left(16 \pi^{2}\right)^{2} s_{W}^{5}} \frac{m_{H}^{2}}{M_{W}^{3}}\left\{+\left(k_{2}^{\mu_{2}} k_{2}^{\mu_{3}}-k_{3}^{\mu_{2}} k_{3}^{\mu_{3}}\right)\left(-\frac{5}{36}-\frac{1}{64} \pi \sqrt{3}+\frac{23}{864} \pi^{2}\right)\right. \\
& \left.+g^{\mu_{2} \mu_{3}}\left(k_{2} \cdot k_{2}-k_{3} \cdot k_{3}\right)\left(-\frac{133}{576}+\frac{1}{64} \pi \sqrt{3}+\frac{5}{432} \pi^{2}\right)\right\} \\
& \frac{\delta \Gamma_{e f f}}{\delta w^{+}\left(k_{1}\right) \delta W_{\mu_{2}}^{-}\left(k_{2}\right) \delta A_{\mu_{3}}\left(k_{3}\right)}=i \frac{e^{5}}{\left(16 \pi^{2}\right)^{2} s_{W}^{4}} \frac{m_{H}^{2}}{M_{W}^{3}}\left\{+k_{2}^{\mu_{2}}\left(k_{2}^{\mu_{3}}-k_{1}^{\mu_{3}}\right)\left(-\frac{71}{192}+\frac{11}{288} \pi^{2}\right)\right. \\
& +k_{2}^{\mu_{3}} k_{3}^{\mu_{2}}\left(-\frac{7}{12}+\frac{1}{18} \pi^{2}\right)+k_{3}^{\mu_{2}} k_{3}^{\mu_{3}}\left(-\frac{133}{288}+\frac{1}{32} \pi \sqrt{3}+\frac{5}{216} \pi^{2}\right) \\
& +g^{\mu_{2} \mu_{3}} k_{2} \cdot k_{2}\left(-\frac{7}{24}+\frac{1}{36} \pi^{2}\right)+g^{\mu_{2} \mu_{3}} k_{1} \cdot k_{1}\left(-\frac{5}{64}+\frac{1}{96} \pi^{2}\right) \\
& \left.+g^{\mu_{2} \mu_{3}} k_{3} \cdot k_{3}\left(\frac{49}{288}-\frac{1}{32} \pi \sqrt{3}+\frac{1}{216} \pi^{2}\right)\right\} \\
& \frac{\delta \Gamma_{e f f}}{\delta w^{+}\left(k_{1}\right) \delta W_{\mu_{2}}^{-}\left(k_{2}\right) \delta Z_{\mu_{3}}\left(k_{3}\right)}=-\frac{s_{W}}{c_{W}} \frac{\delta \Gamma_{\text {eff }}}{\delta w^{+}\left(k_{1}\right) \delta W_{\mu_{2}}^{-}\left(k_{2}\right) \delta A_{\mu_{3}}\left(k_{3}\right)}+\frac{1}{c_{W}} \frac{\delta \Gamma_{e f f}}{\delta z\left(k_{1}\right) \delta W_{\mu_{2}}^{+}\left(k_{2}\right) \delta W_{\mu_{3}}^{-}\left(k_{3}\right)} \\
& +i \frac{e^{5}}{\left(16 \pi^{2}\right)^{2} c_{W}^{3} s_{W}^{3}} \frac{m_{H}^{2}}{M_{W}} g^{\mu_{2} \mu_{3}}\left(\frac{9}{64} \pi \sqrt{3}+\frac{3}{64} \pi^{2}-\frac{9}{16} C l \sqrt{3}-\frac{21}{128}\right) .
\end{aligned}
$$

As one can see from (4.18), the electric charge of the $W^{ \pm}$boson is not renormalized and only the anomalous magnetic moment and the longitudinal tensor, which is zero if both $W$ bosons are physical, appear. For the $Z W^{+} W^{-}$vertex both coupling finite constant renormalization and anomalous magnetic moment interaction terms are present. The results for the $\gamma W^{+} W^{-}, Z W^{+} W^{-}$vertices are equivalent to the results of Ref. [22] and give the same results for the anomalous magnetic moments and corrections to the reaction $e^{+} e^{-} \rightarrow$ $W^{+} W^{-}$. 
And finally the following quartic electroweak vector boson vertices were obtained:

$$
\begin{aligned}
& \frac{\delta \Gamma_{e f f}}{\delta W_{\mu_{1}}^{+}\left(k_{1}\right) \delta W_{\mu_{2}}^{-}\left(k_{2}\right) \delta W_{\mu_{3}}^{+}\left(k_{3}\right) \delta W_{\mu_{4}}^{-}\left(k_{4}\right)}=\frac{e^{6}}{\left(16 \pi^{2}\right)^{2} s_{W}^{6}} \frac{m_{H}^{2}}{M_{W}^{2}}\left\{\left(g^{\mu_{1} \mu_{2}} g^{\mu_{3} \mu_{4}}+g^{\mu_{1} \mu_{4}} g^{\mu_{2} \mu_{3}}\right)\right. \\
& \times\left(+\frac{4027}{768}-\frac{39}{16} \pi C l+\frac{127}{64} \pi \sqrt{3}-\frac{3319}{3456} \pi^{2}-\frac{289}{96} C l \sqrt{3}+\frac{63}{16} \zeta(3)\right) \\
& \left.+g^{\mu_{1} \mu_{3}} g^{\mu_{2} \mu_{4}}\left(+\frac{17}{384}-\frac{11}{16} \pi \sqrt{3}-\frac{23}{1728} \pi^{2}+\frac{97}{48} C l \sqrt{3}\right)\right\} \\
& \approx \frac{e^{6}}{\left(16 \pi^{2}\right)^{2} s_{W}^{6}} \frac{m_{H}^{2}}{M_{W}^{2}}\left\{-1.76815\left(g^{\mu_{1} \mu_{2}} g^{\mu_{3} \mu_{4}}+g^{\mu_{1} \mu_{4}} g^{\mu_{2} \mu_{3}}\right)-0.275572 g^{\mu_{1} \mu_{3}} g^{\mu_{2} \mu_{4}}\right\} \text {, } \\
& \frac{\delta \Gamma_{e f f}}{\delta W_{\mu_{1}}^{+}\left(k_{1}\right) \delta W_{\mu_{2}}^{-}\left(k_{2}\right) \delta Z_{\mu_{3}}\left(k_{3}\right) \delta Z_{\mu_{4}}\left(k_{4}\right)}=\frac{e^{6}}{\left(16 \pi^{2}\right)^{2} s_{W}^{6} c_{W}^{2}} \frac{m_{H}^{2}}{M_{W}^{2}}\{ \\
& +g^{\mu_{1} \mu_{2}} g^{\mu_{3} \mu_{4}}\left[+\frac{5147}{1152}-\frac{39}{16} \pi C l+\frac{109}{64} \pi \sqrt{3}-\frac{139}{144} \pi^{2}-\frac{85}{48} C l \sqrt{3}+\frac{63}{16} \zeta(3)\right. \\
& \left.+c_{W}^{2}\left(+\frac{217}{288}+\frac{5}{8} \pi \sqrt{3}+\frac{5}{432} \pi^{2}-\frac{9}{4} C l \sqrt{3}\right)\right] \\
& +\left(g^{\mu_{1} \mu_{3}} g^{\mu_{2} \mu_{4}}+g^{\mu_{1} \mu_{4}} g^{\mu_{2} \mu_{3}}\right)\left[+\frac{67}{2304}-\frac{1}{32} \pi \sqrt{3}+\frac{43}{1152} \pi^{2}-\frac{11}{96} C l \sqrt{3}\right. \\
& \left.\left.+c_{W}^{2}\left(+\frac{209}{576}-\frac{5}{16} \pi \sqrt{3}-\frac{71}{864} \pi^{2}+\frac{9}{8} C l \sqrt{3}\right)+c_{W}^{4}\left(-\frac{71}{192}+\frac{11}{288} \pi^{2}\right)\right]\right\} \\
& \approx \frac{e^{6}}{\left(16 \pi^{2}\right)^{2} s_{W}^{6} c_{W}^{2}} \frac{m_{H}^{2}}{M_{W}^{2}}\left\{+g^{\mu_{1} \mu_{2}} g^{\mu_{3} \mu_{4}}\left[-1.94360+0.313234 c_{W}^{2}\right]\right. \\
& \left.+\left(g^{\mu_{1} \mu_{3}} g^{\mu_{2} \mu_{4}}+g^{\mu_{1} \mu_{4}} g^{\mu_{2} \mu_{3}}\right)\left[2.60033 \times 10^{-2}-0.170962 c_{W}^{2}+7.17239 \times 10^{-3} c_{W}^{4}\right]\right\} \\
& \frac{\delta \Gamma_{e f f}}{\delta Z_{\mu_{1}}\left(k_{1}\right) \delta Z_{\mu_{2}}\left(k_{2}\right) \delta Z_{\mu_{3}}\left(k_{3}\right) \delta Z_{\mu_{4}}\left(k_{4}\right)}= \\
& \frac{e^{6}}{\left(16 \pi^{2}\right)^{2} s_{W}^{6} c_{W}^{4}} \frac{m_{H}^{2}}{M_{W}^{2}}\left(g^{\mu_{1} \mu_{2}} g^{\mu_{3} \mu_{4}}+g^{\mu_{1} \mu_{3}} g^{\mu_{2} \mu_{4}}+g^{\mu_{1} \mu_{4}} g^{\mu_{2} \mu_{3}}\right) \\
& \times\left(+\frac{337}{64}-\frac{39}{16} \pi C l+\frac{105}{64} \pi \sqrt{3}-\frac{557}{576} \pi^{2}-2 C l \sqrt{3}+\frac{63}{16} \zeta(3)\right) \\
& \approx-1.90594 \frac{e^{6}}{\left(16 \pi^{2}\right)^{2} s_{W}^{6} c_{W}^{4}} \frac{m_{H}^{2}}{M_{W}^{2}}\left(g^{\mu_{1} \mu_{2}} g^{\mu_{3} \mu_{4}}+g^{\mu_{1} \mu_{3}} g^{\mu_{2} \mu_{4}}+g^{\mu_{1} \mu_{4}} g^{\mu_{2} \mu_{3}}\right) \\
& \frac{\delta \Gamma_{e f f}}{\delta A_{\mu_{1}}\left(k_{1}\right) \delta A_{\mu_{2}}\left(k_{2}\right) \delta W_{\mu_{3}}^{+}\left(k_{3}\right) \delta W_{\mu_{4}}^{-}\left(k_{4}\right)}= \\
& \frac{e^{6}}{\left(16 \pi^{2}\right)^{2} s_{W}^{4}} \frac{m_{H}^{2}}{M_{W}^{2}}\left(g^{\mu_{1} \mu_{3}} g^{\mu_{2} \mu_{4}}+g^{\mu_{1} \mu_{4}} g^{\mu_{2} \mu_{3}}\right)\left(-\frac{71}{192}+\frac{11}{288} \pi^{2}\right) \\
& \approx 7.17239 \times 10^{-3} \frac{e^{6}}{\left(16 \pi^{2}\right)^{2} s_{W}^{4}} \frac{m_{H}^{2}}{M_{W}^{2}}\left(g^{\mu_{1} \mu_{3}} g^{\mu_{2} \mu_{4}}+g^{\mu_{1} \mu_{4}} g^{\mu_{2} \mu_{3}}\right) \text {, }
\end{aligned}
$$




$$
\begin{aligned}
& \frac{\delta \Gamma_{\text {eff }}}{\delta A_{\mu_{1}}\left(k_{1}\right) \delta Z_{\mu_{2}}\left(k_{2}\right) \delta W_{\mu_{3}}^{+}\left(k_{3}\right) \delta W_{\mu_{4}}^{-}\left(k_{4}\right)}= \\
& \frac{e^{6}}{\left(16 \pi^{2}\right)^{2} s_{W}^{5} c_{W}} \frac{m_{H}^{2}}{M_{W}^{2}}\left\{+g^{\mu_{1} \mu_{2}} g^{\mu_{3} \mu_{4}}\left(+\frac{217}{576}+\frac{5}{16} \pi \sqrt{3}+\frac{5}{864} \pi^{2}-\frac{9}{8} C l \sqrt{3}\right)\right. \\
& \left.+\left(g^{\mu_{1} \mu_{3}} g^{\mu_{2} \mu_{4}}+g^{\mu_{1} \mu_{4}} g^{\mu_{2} \mu_{3}}\right)\left[+\frac{209}{1152}-\frac{5}{32} \pi \sqrt{3}-\frac{71}{1728} \pi^{2}+\frac{9}{16} C l \sqrt{3}+c_{W}^{2}\left(-\frac{71}{192}+\frac{11}{288} \pi^{2}\right)\right]\right\} \\
& \approx \frac{e^{6}}{\left(16 \pi^{2}\right)^{2} s_{W}^{5} c_{W}} \frac{m_{H}^{2}}{M_{W}^{2}}\left\{+0.156617 g^{\mu_{1} \mu_{2}} g^{\mu_{3} \mu_{4}}\right. \\
& \left.+\left(g^{\mu_{1} \mu_{3}} g^{\mu_{2} \mu_{4}}+g^{\mu_{1} \mu_{4}} g^{\mu_{2} \mu_{3}}\right)\left[-8.54809 \times 10^{-2}+7.17239 \times 10^{-3} c_{W}^{2}\right]\right\} \\
& \frac{\delta \Gamma_{e f f}}{\delta A_{\mu_{1}}\left(k_{1}\right) \delta A_{\mu_{2}}\left(k_{2}\right) \delta A_{\mu_{3}}\left(k_{3}\right) \delta A_{\mu_{4}}\left(k_{4}\right)}=\frac{\delta \Gamma_{e f f}}{\delta A_{\mu_{1}}\left(k_{1}\right) \delta A_{\mu_{2}}\left(k_{2}\right) \delta Z_{\mu_{3}}\left(k_{3}\right) \delta Z_{\mu_{4}}\left(k_{4}\right)}=\ldots=0 .
\end{aligned}
$$

There is a $Z Z Z Z$ vertex, which is not present in the $\mathcal{S M}$ at the tree-level. All the other vertices with neutral gauge bosons and at least one photon vanish. Also the $\gamma \gamma W^{+} W^{-}$quartic coupling is generated. Obviously, the tensor structures proportional to $\left(-71 / 192+\pi^{2} 11 / 288\right)$, explicitly appearing in Eq. (4.26) and the expressions for the two-point functions (4.11)-(4.16), are different terms entering the chiral Lagrangian $\mathcal{L}_{11}$ [29]

$$
\mathcal{L}_{11}=\operatorname{Tr}\left[\mathcal{D}_{\mu} \mathcal{V}^{\mu} \mathcal{D}_{\nu} \mathcal{V}^{\nu}\right]
$$

where the Nambu-Goldstone bosons $\omega^{i}$ are assembled in a unitary matrix $\Sigma=\exp \left(i \omega^{i} \tau^{i} / v\right)$ and

$$
\mathcal{D}_{\mu} \Sigma=\partial_{\mu} \Sigma+\frac{i}{2}\left(g \hat{W}_{\mu} \Sigma-g^{\prime} \Sigma \tau_{3} B_{\mu}\right) ; \quad \mathcal{V}_{\mu}=\left(\mathcal{D}_{\mu} \Sigma\right) \Sigma^{\dagger} .
$$

As one can see from (4.6)-(4.8), (4.18)-(4.19) the two-loop $m_{H}^{2}$ corrections to fermion scattering processes and triple vector boson couplings are very small, inspite of the $m_{H}^{2} / M_{W}^{2}$ enhancements, not only because of the small two-loop factor $g^{4} /\left(16 \pi^{2}\right)^{2}$, but also because the dimensionless coefficients themselves are quite small. E.g., the largest coefficient that enters the expression for $s_{W \text { eff }}^{2}(4.8)$ is approximately 0.1. The typical values for other dimensionless coefficients are several units times $10^{-2}$. In this respect the $W^{+} W^{-} W^{+} W^{-}$, $W^{+} W^{-} Z Z$ and $Z Z Z Z$ quartic couplings represent a drastic contrast to the other vertices. The dimensionless coefficients in (4.23), 4.24), (4.25) are about 2, i.e. about 20 times larger, than for $s_{W \text { eff }}^{2}$ (4.8)! As was mentioned previously, these particular vertices are distinguished, due to a contribution from two-loop Higgs self energy insertion in the Higgs-reducible graphs. These vertices receive a contribution from the $\zeta(3)$ and $\pi C l$ terms, which originate only from the two-loop Higgs mass counterterm 8] as a term proportional to a linear combination $21 \zeta(3)-13 \pi C l$. In a sense these couplings could be considered "genuine" quartic couplings, which are the most sensitive to the details of the mechanism of the electroweak symmetry breaking.

\section{Numerical results}

The possibilities to probe the quartic vector boson couplings through the $W W$-, $Z Z$-fusion reactions (4.1) [30] and through $W W Z / Z Z Z$ triple gauge boson production (4.2) [31] at high energy linear colliders are under intense study. In this Section we will not give any extensive phenomenological analyses, but present cross-sections and distributions for the fundamental subprocesses of electroweak gauge boson scattering reactions:

$$
V V \rightarrow V V
$$

In Fig. 3 we show angular distributions for processes of type (5.1). We present these distributions both for unpolarized and longitudinal vector bosons. The energy is taken to be $\sqrt{s_{V V}}=500 \mathrm{GeV}$ and the Higgs mass $m_{H}=900 \mathrm{GeV}$. We give the results both for the tree-level and for the tree level plus the two-loop $m_{H}^{2}$ 

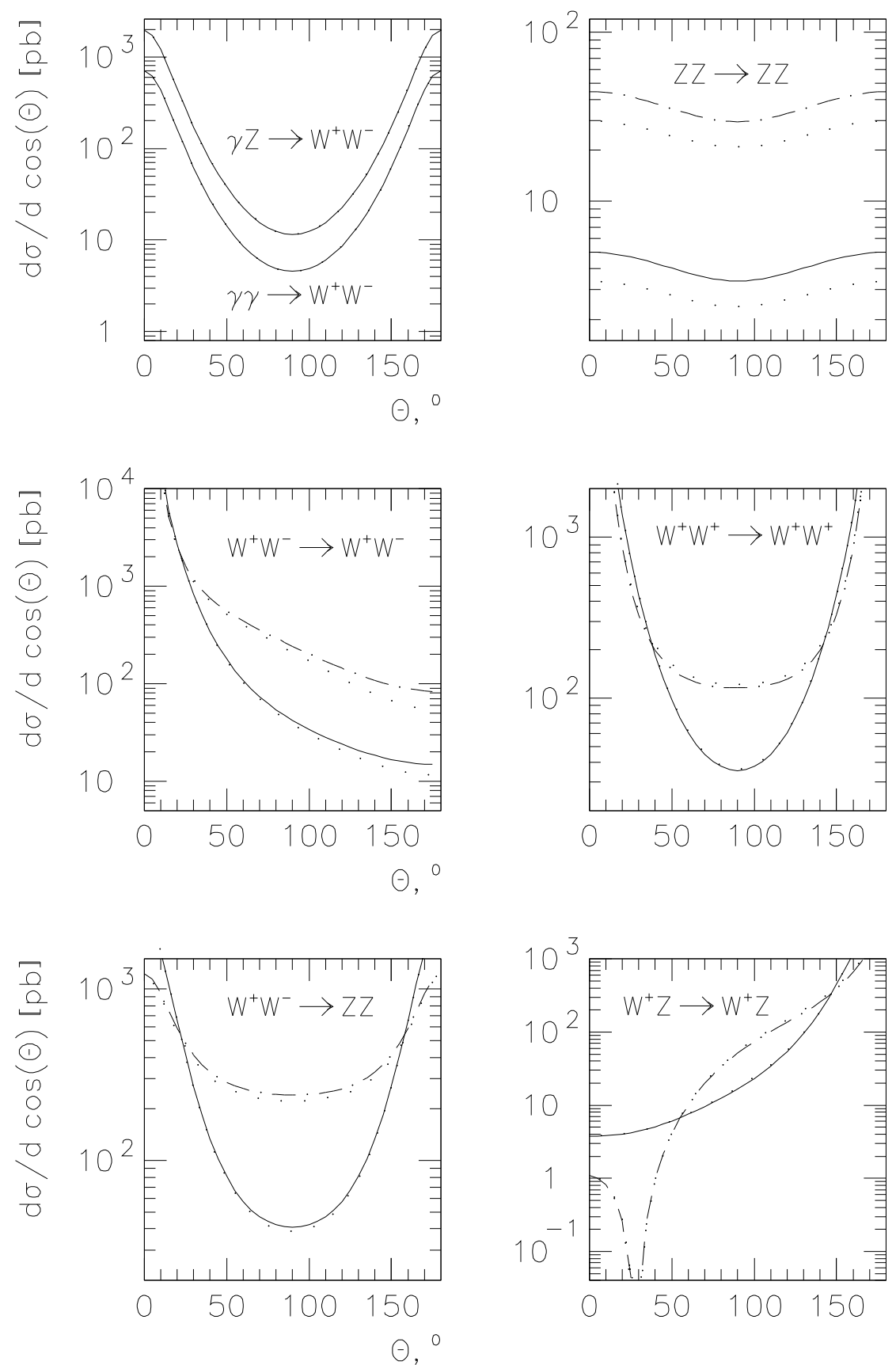

Figure 3: Born differential cross sections for unpolarized $U U U U$ (solid line) and longitudinal $L L L L$ (dotdashed line) vector bosons at $\sqrt{s_{V V}}=500 \mathrm{GeV}$ and $m_{H}=900 \mathrm{GeV}$. Dotted lines lines show corresponding two-loop corrected cross sections. 

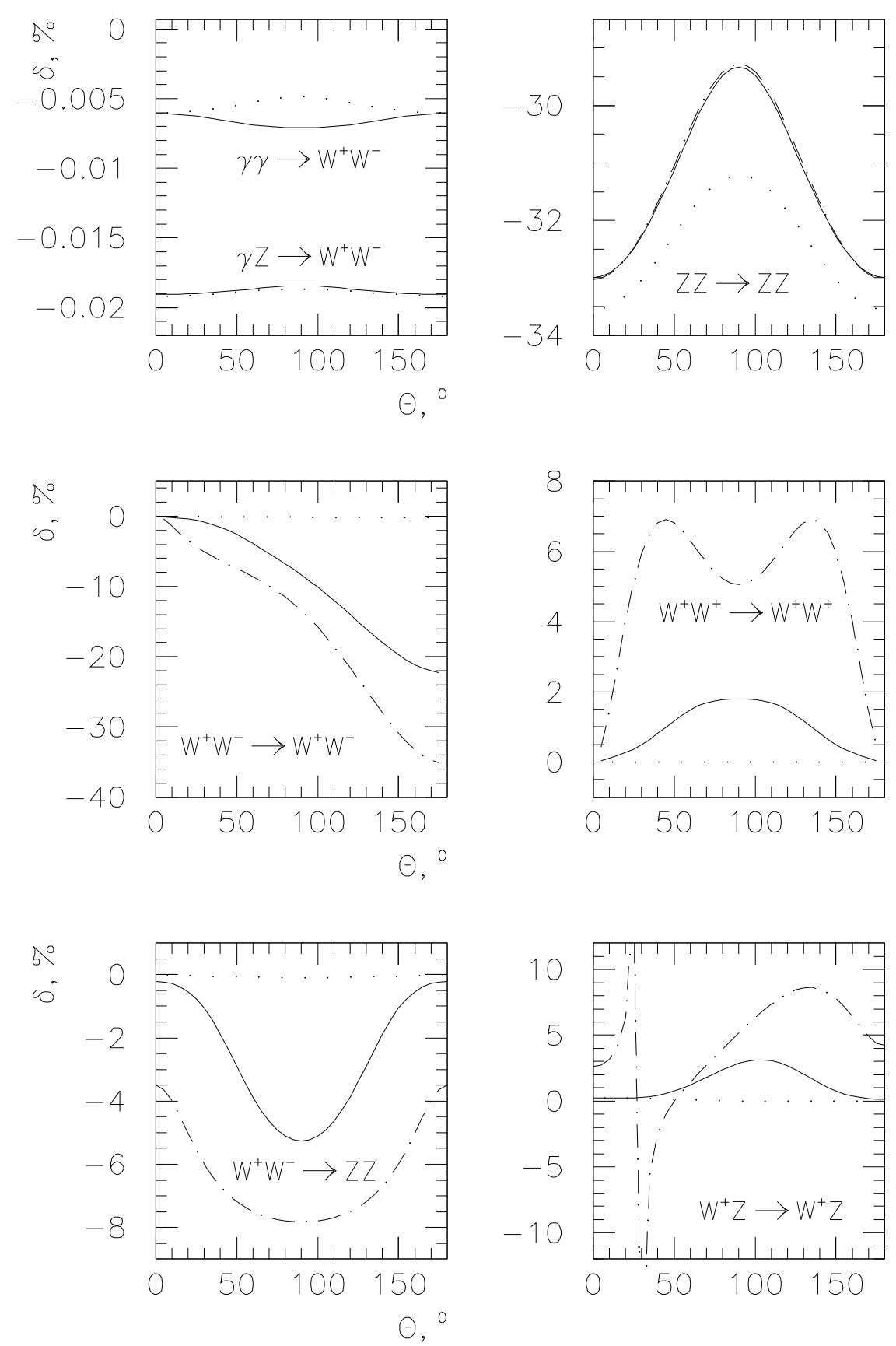

Figure 4: Relative corrections to the Born cross sections in Fig. 3. Solid line denotes correction for unpolarized case $U U U U$, dash-dotted for the longitudinal $L L L L$ polarization, and dotted line for the transverse TTTT polarization. 
Table 1: Differential $d \sigma / d \cos (\theta)$ and total Born cross sections and relative corrections for unpolarized, purely longitudinal $L L L L$ and purely transverse $T T T T$ vector boson scattering at the center-of-mass energy $\sqrt{s}=500 \mathrm{GeV}$ and $m_{H}=900 \mathrm{GeV}$.

\begin{tabular}{|c|c|c|c|c|c|c|}
\hline$\gamma \gamma \rightarrow W^{+} W^{-}$ & $\sigma^{u n p o l}, p b$ & $\delta^{\text {unpol }}, \%$ & $\sigma^{L L L L}, p b$ & $\delta^{L L L L}, \%$ & $\sigma^{T T T T}, p b$ & $\delta^{T T T T}, \%$ \\
\hline $30^{\circ}$ & 61.97 & $-6.34 \cdot 10^{-3}$ & - & - & 60.94 & $-5.84 \cdot 10^{-3}$ \\
\hline $60^{\circ}$ & 8.598 & $-6.87 \cdot 10^{-3}$ & - & - & 7.995 & $-5.28 \cdot 10^{-3}$ \\
\hline $90^{\circ}$ & 4.551 & $-7.09 \cdot 10^{-3}$ & 一 & - & 4.044 & $-4.86 \cdot 10^{-3}$ \\
\hline $0^{\circ}<\theta<180^{\circ}$ & 77.48 & $-6.30 \cdot 10^{-3}$ & - & - & 76.10 & $-5.84 \cdot 10^{-3}$ \\
\hline$\overline{\gamma \gamma Z \rightarrow W^{+} W^{-}}$ & $\overline{\sigma^{u n p o l}, p b}$ & $\overline{\delta^{\text {unpol }}, \%}$ & $\overline{\sigma^{L L L L}, p b}$ & $\overline{\delta^{L L L L}, \%}$ & $\overline{\sigma^{T T T T}, p b}$ & $\overline{\delta^{T T T T}, \%}$ \\
\hline $30^{\circ}$ & 171.0 & $-1.90 \cdot 10^{-2}$ & - & - & 219.4 & $-1.91 \cdot 10^{-2}$ \\
\hline $60^{\circ}$ & 22.66 & $-1.87 \cdot 10^{-2}$ & - & - & 28.76 & $-1.88 \cdot 10^{-2}$ \\
\hline $90^{\circ}$ & 11.48 & $-1.84 \cdot 10^{-2}$ & - & - & 14.54 & $-1.87 \cdot 10^{-2}$ \\
\hline $0^{\circ}<\theta<180^{\circ}$ & 213.1 & $-1.90 \cdot 10^{-2}$ & - & - & 274.0 & $-1.91 \cdot 10^{-2}$ \\
\hline 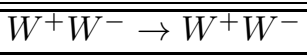 & $\overline{\sigma^{\text {unpol }}, p b}$ & $\delta^{\text {unpol }, \%}$ & $\overline{\sigma^{L L L L}, p b}$ & $\delta^{L L L L}, \%$ & 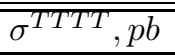 & $\bar{C}^{T T T T}, \%$ \\
\hline $30^{\circ}$ & 828.0 & -0.832 & 1176. & -5.05 & 1163. & $-4.50 \cdot 10^{-2}$ \\
\hline $60^{\circ}$ & 108.1 & -3.87 & 441.0 & -8.58 & 94.56 & $-8.85 \cdot 10^{-2}$ \\
\hline $90^{\circ}$ & 42.08 & -8.36 & 243.2 & -13.4 & 21.74 & -0.131 \\
\hline $120^{\circ}$ & 23.90 & -14.0 & 144.1 & -21.6 & 11.85 & -0.157 \\
\hline $150^{\circ}$ & 16.73 & -19.7 & 96.91 & -30.9 & 10.57 & -0.178 \\
\hline $10^{\circ}<\theta<170^{\circ}$ & 572.1 & -1.46 & 939.7 & -8.01 & 731.0 & $-3.82 \cdot 10^{-2}$ \\
\hline$\overline{W^{+} W^{+} \rightarrow W^{+} W^{+}}$ & $\overline{\sigma^{u n p o l}, p b}$ & $\overline{\delta^{u n p o l}, \%}$ & $\overline{\sigma^{L L L L}, p b}$ & $\overline{\delta^{L L L L}, \%}$ & $\overline{\sigma^{T T T T}, p b}$ & $\overline{\delta^{T T T T}, \%}$ \\
\hline $30^{\circ}$ & 435.4 & 0.496 & 327.9 & 5.94 & 695.9 & $-5.71 \cdot 10^{-3}$ \\
\hline $60^{\circ}$ & 61.07 & 1.49 & 130.9 & 6.31 & 86.13 & $+6.02 \cdot 10^{-3}$ \\
\hline $90^{\circ}$ & 35.44 & 1.79 & 115.7 & 5.05 & 43.15 & $+3.30 \cdot 10^{-3}$ \\
\hline $10^{\circ}<\theta<170^{\circ}$ & 573.8 & 0.456 & 526.4 & 4.54 & 845.2 & $-6.40 \cdot 10^{-3}$ \\
\hline$\overline{W^{+} W^{-} \rightarrow Z Z}$ & $\sigma^{\text {unpol }}, p b$ & $\overline{\delta^{\text {unpol }}, \%}$ & $\overline{\sigma^{L L L L}, p b}$ & $\bar{\delta}^{L L L L}, \%$ & $\sigma^{T T T T}, p b$ & $\bar{\delta}^{T T T T}, \%$ \\
\hline $30^{\circ}$ & 271.2 & -1.06 & 412.6 & -5.99 & 390.7 & $-4.50 \cdot 10^{-2}$ \\
\hline $60^{\circ}$ & 57.85 & -3.87 & 262.7 & -7.49 & 51.47 & $-7.31 \cdot 10^{-2}$ \\
\hline $90^{\circ}$ & 40.75 & -5.26 & 241.1 & -7.82 & 25.83 & $-9.55 \cdot 10^{-2}$ \\
\hline $0^{\circ}<\theta<180^{\circ}$ & 347.1 & -1.43 & 643.5 & -6.66 & 477.9 & $-4.53 \cdot 10^{-2}$ \\
\hline$\overline{W^{+} Z \rightarrow W^{+} Z}$ & $\overline{\sigma^{u n p o l}, p b}$ & $\overline{\delta^{\text {unpol }}, \%}$ & $\overline{\sigma^{L L L L}, p b}$ & 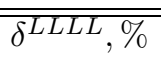 & $\overline{\sigma^{T T T T}, p b}$ & $\bar{\delta}^{T T T T}, \%$ \\
\hline $30^{\circ}$ & 4.417 & 0.275 & $1.79 \cdot 10^{-2}$ & -22.4 & 6.256 & 0.204 \\
\hline $60^{\circ}$ & 7.506 & 1.21 & 10.13 & 1.43 & 7.048 & 0.108 \\
\hline $90^{\circ}$ & 16.76 & 2.85 & 51.19 & 5.17 & 13.05 & $+1.63 \cdot 10^{-2}$ \\
\hline $120^{\circ}$ & 53.96 & 2.68 & 136.1 & 8.15 & 56.89 & $-1.07 \cdot 10^{-2}$ \\
\hline $150^{\circ}$ & 445.1 & 0.765 & 388.3 & 7.77 & 657.2 & $-2.16 \cdot 10^{-2}$ \\
\hline $0^{\circ}<\theta<180^{\circ}$ & 281.0 & 0.718 & 239.2 & 6.81 & 414.7 & $-2.17 \cdot 10^{-2}$ \\
\hline$\overline{Z Z Z \rightarrow Z Z}$ & $\overline{\sigma^{u n p o l}, p b}$ & $\overline{\delta^{u n p o l}, \%}$ & $\overline{\sigma^{L L L L}, p b}$ & $\overline{\delta^{L L L L}, \%}$ & $\overline{\sigma^{T T T T}, p b}$ & $\overline{c \delta^{T T T T}, \%}$ \\
\hline $30^{\circ}$ & 4.570 & -32.3 & 40.41 & -32.2 & $1.41 \cdot 10^{-3}$ & -33.0 \\
\hline $60^{\circ}$ & 3.758 & -30.5 & 32.97 & -30.4 & $1.08 \cdot 10^{-3}$ & -31.8 \\
\hline $90^{\circ}$ & 3.372 & -29.3 & 29.56 & -29.3 & $9.47 \cdot 10^{-4}$ & -31.2 \\
\hline $0^{\circ}<\theta<180^{\circ}$ & 7.799 & -30.8 & 68.60 & -30.8 & $2.29 \cdot 10^{-3}$ & -32.2 \\
\hline
\end{tabular}



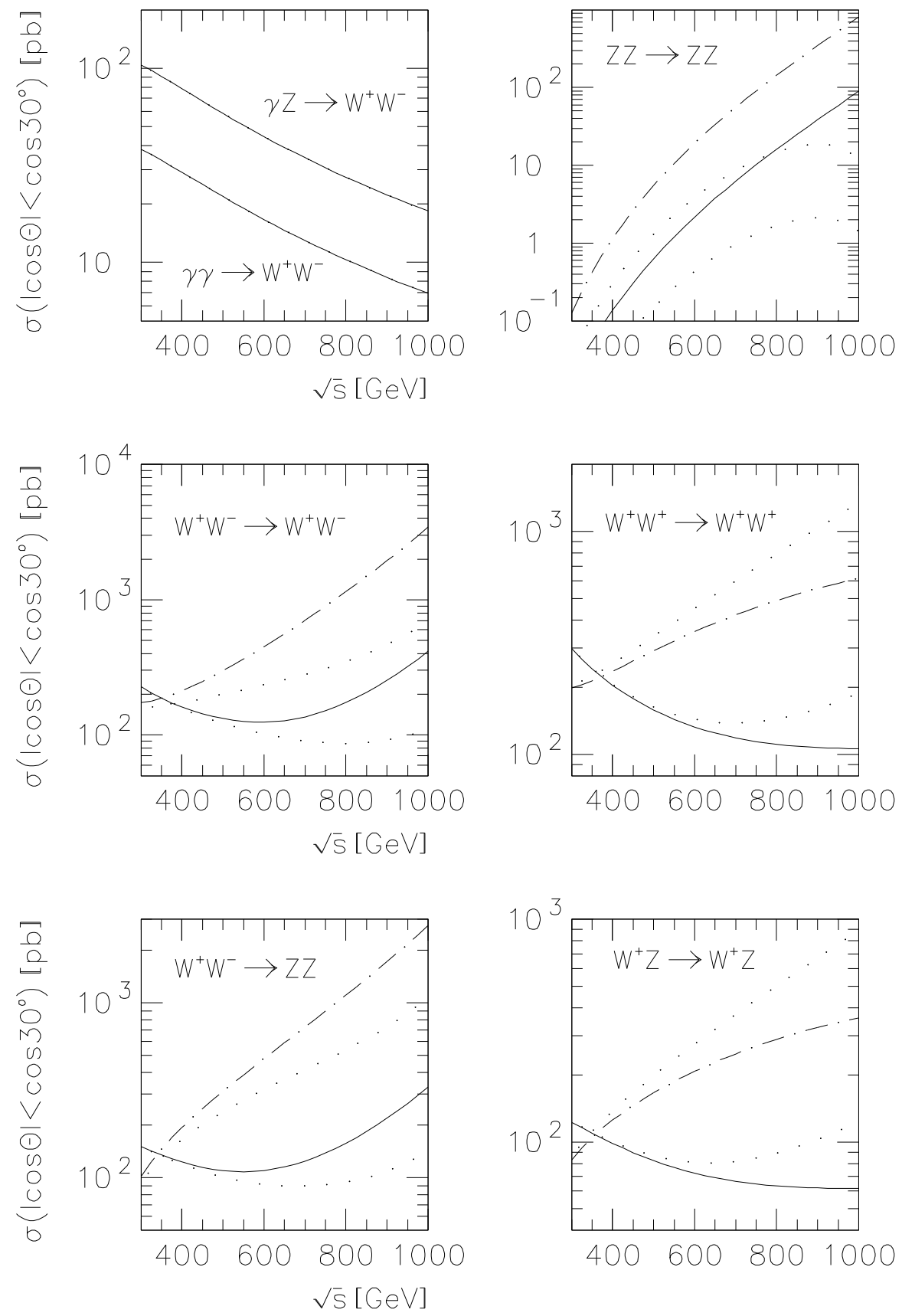

Figure 5: Energy dependence of the cross sections for unpolarized $U U U U$ (solid lines) and longitudinal $L L L L$ (dash-dotted lines) vector boson scattering reactions. Dotted lines show corresponding corrected cross sections. Higgs mass is taken to be $1.5 \mathrm{TeV}$ 
corrections. As one would expect, the largest relative corrections are seen for the $Z Z \rightarrow Z Z$ reaction, which receives only a small contribution from heavy Higgs boson exchange at the tree level.

Relative corrections to the Born cross sections

$$
\delta=\frac{\sigma^{c o r r}-\sigma^{\text {Born }}}{\sigma^{\text {Born }}}
$$

are shown in Fig. 4. As one can see the corrections to the $\gamma \gamma \rightarrow W^{+} W^{-}, \gamma Z \rightarrow W^{+} W^{-}$reactions are at the level of $10^{-2} \%$ and so are unobservably small. Corrections to the other reactions are of the order of $(10-50) \%$, i.e. quite large, especially in some regions of phase space. Corrections for longitudinal polarization $L L L L$ dominate, while corrections for transverse polarization $T T T T$ are negligible for all reactions except $Z Z \rightarrow Z Z$. Corrections for the neutral channel reactions are negative, while for the charged channels $W^{+} W^{+} \rightarrow W^{+} W^{+}, W^{+} Z \rightarrow W^{+} Z$ they are positive. The correction to the reaction $W^{+} Z \rightarrow W^{+} Z$ for $L L L L$ polarization exhibits a pole behavior at $\theta \approx 28^{\circ}$, due to the fact that longitudinal Born cross section is exactly equal to zero at this value of $\theta$.

Differential cross sections at several angles, integrated cross sections and relative corrections are presented in Table 1. Since complete one-loop $\mathcal{S} \mathcal{M}$ electroweak corrections are known for the reactions $\gamma \gamma \rightarrow W^{+} W^{-}$ [32], $Z Z \rightarrow Z Z$ [33] and $W^{+} W^{-} \rightarrow W^{+} W^{-}$[34], one can compare our two-loop $m_{H}^{2}$ corrections with these one-loop results.

One-loop electroweak corrections to the cross section of the $W^{+} W^{-}$-pair production in photon-photon collisions are about $-(1-3) \%$ at $500 \mathrm{GeV}$, so that there is no chance to separate the $m_{H}^{2}$ effects from all other corrections in this reaction.

One-loop corrections to the $W^{+} W^{-} \rightarrow W^{+} W^{-}$and $Z Z \rightarrow Z Z$ scattering reactions for longitudinal $L L L L$ polarization at $500 \mathrm{GeV}$ are positive and of the order of $10 \%$ and $(30-40) \%$, respectively. So the two-loop large Higgs mass corrections proportional to $m_{H}^{2}$ for these reactions are approximately the same in size as one-loop electroweak corrections, but opposite in sign, almost canceling out in the complete amplitude. It means that in order to experimentally extract physically interesting two-loop $m_{H}^{2}$ quantum corrections from the cross sections of vector boson scattering reactions one should necessarily include all one-loop electroweak radiative corrections. Since enhanced two-loop corrections are approximately of the same size as one-loop corrections, experimental accuracy which is enough to measure the effects of oneloop corrections is simultaneously enough to measure the contribution of the large Higgs mass two-loop corrections.

Finally, in order to demonstrate the potential importance of large Higgs mass corrections at high energies, we present in Fig. 5 the energy dependence of the Born and corrected cross section of vector boson scattering integrated over scattering angles in the region $30^{\circ}<\theta<150^{\circ}$ for $\sqrt{s_{V V}}$ up to $1 \mathrm{TeV}$ for the very heavy Higgs boson mass of $1.5 \mathrm{TeV}$. The existence of a physical Higgs particle with such large mass seems to be excluded due to triviality bounds (see [2] and references therein), and the same conclusion follows from the non-perturbative $1 / N$ approach with the account of next-to-leading corrections 16$]$. We can consider however such a value of the $m_{H}$ as an effective ultraviolet cut-off in the theory without visible scalar Higgs particle. We see that the growth with energy of the longitudinal vector boson scattering cross sections, which is the experimental indication of the existence of heavy Higgs sector and/or strong interactions among longitudinal $W_{L}, Z_{L}$ bosons, is strongly modified by the two-loop $m_{H}^{2}$ corrections. Again at high energy the cross sections of neutral channel reactions are diminished, and those of charged channel reactions are enhanced. Of course at center-of-mass energy of $1 \mathrm{TeV} s_{V V}$ is not very much smaller than $m_{H}^{2}$, which is the condition under which our low-energy effective action was calculated. Nevertheless, we think that the qualitative trend, namely the fact that the account of large Higgs mass corrections at high energy can change the value of the cross section by a large factor of $2-4$, is important for all considerations of the signal from strong scattering of longitudinal vector boson at TeV energy.

In fact using the results of a thorough phenomenological analysis of the effects of anomalous quartic couplings in $e^{ \pm} e^{-}$collisions 30, 31 we can estimate the potential of $\mathrm{TeV} e^{ \pm} e^{-}$linear colliders in investigating the effects of enhanced $m_{H}^{2}$ two-loop corrections more quantitatively. Anomalous quartic couplings are defined in Ref. [30] through the following effective electroweak chiral Lagrangians:

$$
\mathcal{L}_{4}=g^{4} \alpha_{4}\left[\frac{1}{2}\left[\left(W^{+} W^{-}\right)^{2}+\left(W^{+2}\right)\left(W^{-2}\right)\right]+\frac{1}{c_{W}^{2}}\left(W^{+} Z\right)\left(W^{-} Z\right)+\frac{1}{4 c_{W}^{4}} Z^{4}\right],
$$




$$
\mathcal{L}_{5}=g^{4} \alpha_{5}\left[\left(W^{+} W^{-}\right)^{2}+\frac{1}{c_{W}^{2}}\left(W^{+} W^{-}\right) Z^{2}+\frac{1}{4 c_{W}^{4}} Z^{4}\right],
$$

where $g=e / s_{W}$. These operators introduce all possible quartic couplings among the weak gauge bosons, that are compatible with custodial $S U(2)_{c}$ symmetry [29]. Although our complete effective action given in Section 4 does not obey this symmetry and as a consequence can not be described by the combination of operators (5.3), (5.4), the dominating terms which originate from two-loop Higgs self energy insertions in the Higgs reducible graphs have exactly the structure of Lagrangian (5.4). Using our expressions (4.23), (4.24), (4.25) we can calculate the coupling constant $\alpha_{5}$ :

$$
\alpha_{5} \approx-\frac{g^{2}}{\left(16 \pi^{2}\right)^{2}} \frac{m_{H}^{2}}{M_{W}^{2}} .
$$

In our approach the constant $\alpha_{4}$ should be about an order of magnitude smaller. The $90 \%$ bound, based on the hypothesis $\alpha_{5}=0$, obtained by combining the $e^{+} e^{-} \rightarrow \nu_{e} \bar{\nu}_{e} W^{+} W^{-}$and $e^{+} e^{-} \rightarrow \nu_{e} \bar{\nu}_{e} Z Z$ channels is $\left|\alpha_{5}\right| \leq 1.5 \times 10^{-3}$ for $\sqrt{s}=1.6 \mathrm{TeV}$ and integrated luminosity of $500 \mathrm{fb}^{-1}$ [30]. For the Higgs mass of $1.5 \mathrm{TeV}$ the value of $\alpha_{5}$ from Eq. (5.5) is approximately $-6 \times 10^{-3}$ (and $-2 \times 10^{-3}$ for $m_{H}=900 \mathrm{GeV}$ ), which is four times larger than the achievable experimental limit. This comparison is a very good indication that in the case, if a heavy Higgs scenario of the electroweak symmetry breaking is realized in nature, its indirect quantum effects could be measured.

\section{Conclusions}

Owing to the enhanced sensitivity to the heavy Higgs boson sector of the $\mathcal{S} \mathcal{M}$, two-loop large Higgs mass quantum corrections to low energy vertices growing like $m_{H}^{2}$ found continuous interest in the literature, where corrections to weak vector boson propagators [18 20], triple vector boson vertices [22], as well as corrections due to an arbitrary gauge-invariant non-renormalizable potential of a heavy Higgs particle [35] were calculated, but found to be very small and beyond experimental verification. We have completed the existing results by calculating the two-loop $m_{H}^{2}$ correction for $m_{H} \gg M_{W}$ to quartic electroweak boson vertices at low energy, which were the last quantities, which exhibit power $m_{H}^{2}$ enhancement at the two-loop level.

Corrections are found to be especially large for the $W^{+} W^{-} W^{+} W^{-}, W^{+} W^{-} Z Z$ and $Z Z Z Z$ quartic vertices, which receive contribution from two-loop Higgs self-energy graphs. The value of these corrections for vector boson scattering reactions at $\sqrt{s}=500 \mathrm{GeV}$ is found to be of the order of $(5-30) \%$ for cross sections of the longitudinally polarized particles, which are the most sensitive to the $m_{H}^{2}$ effects.

By comparison with the results of phenomenological analysis of the effects of anomalous quartic couplings in the $W W, Z Z$-fusion reactions in $e^{+} e^{-}$collisions [30] we found that the anomalous interactions generated by the two-loop large Higgs mass quantum corrections seem to be large enough to be observable at the TeV energy colliders.

An important point is the validity of the perturbation theory for large values of Higgs mass and selfcouplings. The physical Higgs boson seems to be excluded by purely theoretical reasons at $m_{H}$ above $1 \mathrm{TeV}$ [2]. However, it is quite encouraging that for the Higgs mass below $1 \mathrm{TeV}$ the perturbative two-loop corrections, that are substantial when compared to the one-loop and tree level, turn out to be remarkably close to the nonperturbative results obtained in next-to-leading approximation in $1 / N$ expansion [16].

\section{Acknowledgements}

G.J. is indebted to J.J. van der Bij, G. Degrassi and A. Ghinculov for very useful discussions. This work was supported in part by the Alexander von Humboldt Foundation and the Russian Foundation for Basic Research grant 96-02-19-464. 


\section{References}

[1] J. Timmermans, talk given at the XVIII International Symposium on Lepton and Photon Interactions, 28 July 1997 - 1 August 1997, Hamburg.

[2] G. Altarelli, ibid..

[3] P. Langacker, talk at International Workshop on Supersymmetry and Unification of Fundamental Interactions (SUSY 95), Palaiseau, France, 15-19 May 1995, NSF-ITP-95-140, October 1995, hep$\mathrm{ph} / 9511207$;

W. Hollik, talk at 11th Topical Workshop on Proton-Antiproton Collider Physics (PBARP 96), Padua, Abano Terme, Italy, 26 May - 1 June 1996, Karlsruhe University preprint No KA-TP-19-1996, hep$\mathrm{ph} / 9608325$

G. Passarino, talk at CRAD96, Cracow, August 1996, hep-ph/9604344;

U. Baur and M. Demarteau, in: Proceedings of the DPF/DPB Summer Study, Snowmass 1996.

[4] G. Degrassi, P. Gambino, M. Passera, and A. Sirlin, CERN-TH-97-197, hep-ph/9708311, August 1997.

[5] L. Durand, P.N. Maher and K. Riesselmann, Phys. Rev. D48 (1993) 1084;

K. Riesselmann, Phys. Rev. D53 (1996) 6226;

K. Riesselmann and S. Willenbrock, Phys. Rev. D55 (1997) 311.

[6] A. Ghinculov and J.J. van der Bij, Nucl. Phys. B436 (1995) 30.

[7] P.N. Maher, L. Durand and K. Riesselmann, Phys. Rev. D48 (1993) 1061; D52 (1995) 553 (E).

[8] V. Borodulin, G. Jikia, Phys. Lett. B391 (1997) 434.

[9] A. Ghinculov, T. Binoth, Phys. Lett. B394 (1997) 139.

[10] A. Ghinculov, Phys. Lett. B337 (1994) 137; B346 (1995) 426 (E).

[11] L. Durand, B.A. Kniehl and K. Riesselmann, Phys. Rev. Lett. 72 (1994) 2534; 74 (1995) 1699 (E); Phys. Rev. D51 (1995) 5007.

[12] A. Ghinculov, Nucl. Phys. B455 (1995) 21.

[13] A. Frink, B.A. Kniehl, D. Kreimer and K. Riesselmann, Phys. Rev. D54 (1996) 4548.

[14] A. Ghinculov and J.J. van der Bij, Nucl. Phys. B482 (1996) 59.

[15] T. Binoth, A. Ghinculov, Phys. Rev. D56 (1997) 3147.

[16] A. Ghinculov, T. Binoth, J.J. van der Bij, FREIBURG-THEP-97-19, August 1997, hep-ph/9709211; FREIBURG-THEP-97-20, September 1997, hep-ph/9711318.

[17] M. Veltman, Acta Phys. Pol. B8 (1977) 475; Phys. Lett. B70 (1977) 253; Phys. Lett. B91 (1980) 95.

[18] J. van der Bij, M. Veltman, Nucl. Phys. B231 (1984) 205.

[19] J.J. van der Bij, Nucl. Phys. B248 (1984) 141.

[20] R. Barbieri, P. Ciafaloni, A. Strumia, Phys. Lett. B317 (1993) 381.

[21] M.B. Einhorn, J. Wudka, Phys. Rev. D39 (1989) 2758;Phys. Rev. D47 (1993) 5029.

[22] J.J. van der Bij, Nucl. Phys. B255 (1985) 648.

[23] K.-i. Aoki, Z. Hioki, R. Kawabe, M. Konuma and T. Muta, Suppl. Prog. Theor. Phys. 73 (1982) 1; M. Böhm, H. Spiesberger and W. Hollik, Fortschr. Phys. 34 (1986) 687.

[24] S. Willenbrock and G. Valencia, Phys. Lett. B247 (1990) 341. 
[25] R.G. Stuart, Phys. Lett. B272 (1991) 353.

[26] F.V. Tkachov, Phys. Lett. B124 (1983) 212; Int. J. Mod. Phys. A8 (1993) 2047.

[27] F.V. Tkachov, Phys. Lett. B100 (1981) 65;

K.G. Chetyrkin, F.V. Tkachov, Nucl. Phys. B192 (1981) 159.

[28] B.W. Lee, C. Quigg, and H. Thacker, Phys. Rev. D16 (1977) 1519;

M.S. Chanowitz and M.K. Gaillard, Nucl. Phys. B261 (1985) 379;

G.J. Gounaris, R. Kögerler and H. Neufeld, Phys. Rev. D34 (1986) 3257.

[29] T. Appelquist and C. Bernard, Phys. Rev. D22 (1980) 200;

A. Longhitano, Nucl. Phys. B188 (1981) 118;

A. Falk, M. Luke and E.H. Simmons, Nucl. Phys. B365 (1991) 523;

F. Feruglio, Int. J. Mod. Phys. A8 (1993) 4937;

T. Appelquist and G.H. Wu, Phys. Rev. D48 (1993) 3235;

F. Boudjema, in Proceedings of the Workshop $e^{+} e^{-}$Collisions at $500 \mathrm{GeV}$ : the Physics Potential, Part C, Munich-Annecy-Hamburg, 1993, DESY 93-123C, p. 177.

[30] E. Boos, H.J. He, W. Kilian, A. Pukhov, C.P. Yuan, P.M. Zerwas, DESY-96-256, August 1997, hep$\mathrm{ph} / 9708310$;

T. Han, H.-J. He, C.P. Yuan, UCD-97-22, November 1997, hep-ph/9711429.

[31] A. Ghinculov, J.J. van der Bij Phys. Lett. B279 (1992) 189;

G. Bélanger and F. Boudjema, Phys. Lett. B288 (1992) 210;

S. Dawson, A. Likhoded, G. Valencia, O. Yushchenko, Proceedings of 1996 DPF / DPB Summer Study on New Directions for High-Energy Physics, Snowmass, CO, 25 June - 12 July 1996, hep-ph/9610299;

O.J.P. Éboli, M.C. Gonzalez-Garcia, and J.K. Mizukoshi, IFT-P.079/97, hep-ph/9711499.

[32] A. Denner, S. Dittmaier, and R. Schuster, Nucl. Phys. B452 (1995) 80;

G. Jikia, Nucl. Phys. B494 (1997) 19.

[33] A. Denner, S. Dittmaier, T. Hahn, Phys. Rev. D56 (1997) 117.

[34] A. Denner, T. Hahn, PSI-PR-97-31, November 1997, hep-ph/9711302.

[35] J.J. van der Bij, Nucl. Phys. B267 (1986) 557. 